Active and Passive Elec. Comp., 2002, Vol. 25, pp. 201-209

Taylor \& Francis

Taylor \& Francis Group

\title{
THE STABILIZATION OF NIOBIUM-BASED SOLID ELECTROLYTE CAPACITORS*
}

\author{
YONGJIAN QIU ${ }^{\mathrm{a}}$, DON SMYTH ${ }^{\mathrm{b}}$ and JON KIMMEL ${ }^{\mathrm{a}, \uparrow}$ \\ ${ }^{\mathrm{a} C a b o t ~ P e r f o r m a n c e ~ M a t e r i a l s ~ B o y e r t o w n, ~ P A ~ 19512 ; ~}{ }^{\mathrm{b}}$ Lehigh University, Material Research \\ Center 5E Packer Avenue, PA 18015
}

(Received November 2001)

Keywords: Niobium capacitors, solid electrolyte capacitors, Niobium oxides

\section{INTRODUCTION}

Solid electrolyte tantalum capacitors ( $\mathrm{Ta}$ ) are a major part of the passive electronic components industry. These capacitors are comprised of a sintered porous Ta powder compact on which a dielectric film of tantalum pentoxide $\left(\mathrm{Ta}_{2} \mathrm{O}_{5}\right)$ is grown electrochemically. In most cases, a conducting $\mathrm{MnO}_{2}$ counter-electrode is applied by pyrolysis of an aqueous solution of $\mathrm{Mn}\left(\mathrm{NO}_{3}\right)_{2}$. Such capacitors have very high volumetric efficiency and excellent long-term stability. However, in certain applications, some of the tantalum properties are not required. This has led to the development of high efficiency ceramic capacitors and organic semiconductor aluminum capacitors that can substitute for Ta in some applications. Another potential substitute material is niobium $(\mathrm{Nb})$, which has electrical and chemical properties similar to tantalum. Early attempts to use $\mathrm{Nb}$ failed because of an unstable niobium/niobium pentoxide $\left(\mathrm{Nb}_{2} \mathrm{O}_{5}\right)$ interface [1], but recently two approaches to stabilization have been developed. One is based on the known effect of Ta nitriding, and the other takes advantage of the unique properties of niobium monoxide $(\mathrm{NbO})$. This paper discusses the dynamics of the substrate/dielectric interface and shows how control of oxygen migration at the interface results in improved capacitor performance.

\section{TANTALUM AND NIOBIUM DIELECTRIC BEHAVIOR}

In an ideal capacitor, there is an abrupt transition from a highly conducting electrode to the insulating dielectric. Such a sharp interface is achieved by the electrolytic oxidation of $\mathrm{Ta}$ or $\mathrm{Nb}$

\footnotetext{
* An earlier version of this paper was published in the Proceedings of the 15th Annual European Passive Components Conference (CARTS-EUROPE 2001), 15-19 October 2001, pp. 169-174.

$\dagger$ Tel.: 610-369-8239; Fax: 610-369-0516; E-mail: jonathan_kimmel@cabot_corp.com
} 
to form the dielectric oxide. However, this interface is highly unstable in a thermodynamic sense, and at elevated temperatures, where atomic diffusion becomes possible, the underlying metal extracts oxygen from the dielectric film. When the heat-treatment is done in air, a steady state eventually occurs, with oxygen flowing into the oxide film from the air, diffusing across the film, and then passing into solid solution in the substrate metal [1-3]. This results in a gradient of oxygen deficiency and a gradient of accompanying electrons across the film with the highest value adjacent to the metal. This in turn gives rise to an exponential gradient of semiconductivity decreasing into the film from its highest value at the metal/metal oxide interface. Although the amount of oxygen deficiency is assumed to be very small, and the resulting conductivity is also very small, in the range $10^{-9}-10^{-6} \mathrm{~S} / \mathrm{cm}$, it is sufficient to have a substantial effect on the capacitor properties. In effect the conductivity shorts out some of the dielectric thickness. It has been shown that for this case there is a conductivity level, $\sigma_{0}=\omega \varepsilon \varepsilon_{0}$, such that parts of the film with higher conductivity act as an extension of the electrode, while those parts with conductivity lower than $\sigma_{0}$ act as dielectric ( $\omega$ is $2 \pi f$, where $f$ is the frequency of the ac signal, $\varepsilon$ is the dielectric constant of the oxide, and $\varepsilon_{0}$ is the permittivity of free space, $8.85 \times 10^{-14}$ farads $/ \mathrm{cm}$ ) [3]. A schematic representation of this model is shown in Figure 1, where the log of the local conductivity is plotted against position in the oxide film. The conductivity profile is shown for three temperatures, and the level of $\sigma_{0}$ is shown for one frequency.

The effective dielectric thickness, where $\sigma<\sigma_{0}$, is determined by the intersection of the conductivity profile with $\sigma_{0}$. At constant frequency, the conductivity profile sweeps up and down with temperature over a fixed value of $\sigma_{0}$, with an activation energy of about $0.6 \mathrm{eV}$, while at constant temperature $\sigma_{0}$ sweeps up and down with frequency over a fixed conductivity profile. Thus the measured capacitance defines an effective dielectric thickness, measured from the oxide-electrolyte interface, and $\sigma_{0}$ gives the conductivity at that point in the film. Measurement of the capacitance as a function of frequency then yields the conductivity as a function of position in the dielectric. Plots of such results at various measurement temperature are shown in Figures $2 \mathrm{a}$ and $2 \mathrm{~b}$ for $\mathrm{Ta} / \mathrm{Ta}_{2} \mathrm{O}_{5}$ and $\mathrm{Nb} / \mathrm{Nb}_{2} \mathrm{O}_{5}$, respectively. For

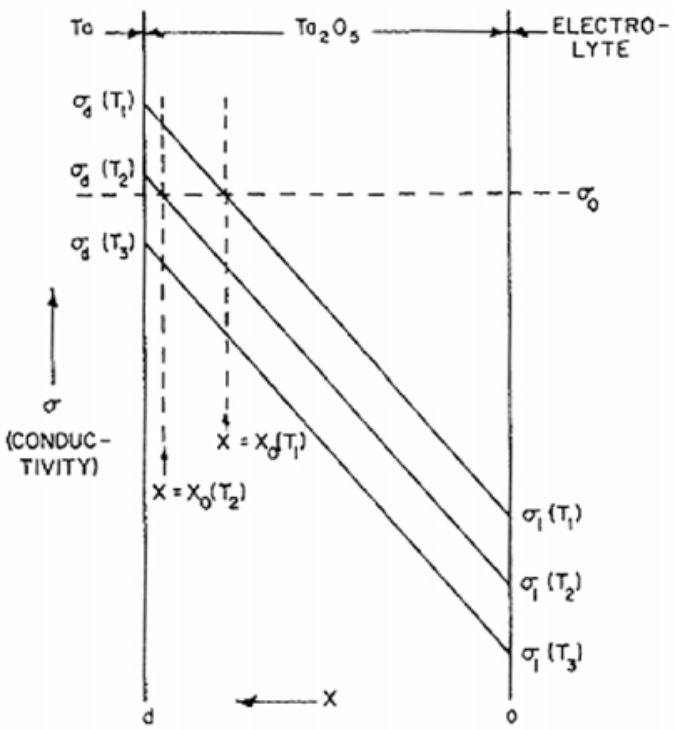

FIGURE 1 Schematic representation of the conductivity profile across heat-treated anodized tantalum at three temperatures, $T_{1}>T_{2}>T_{3}$. 


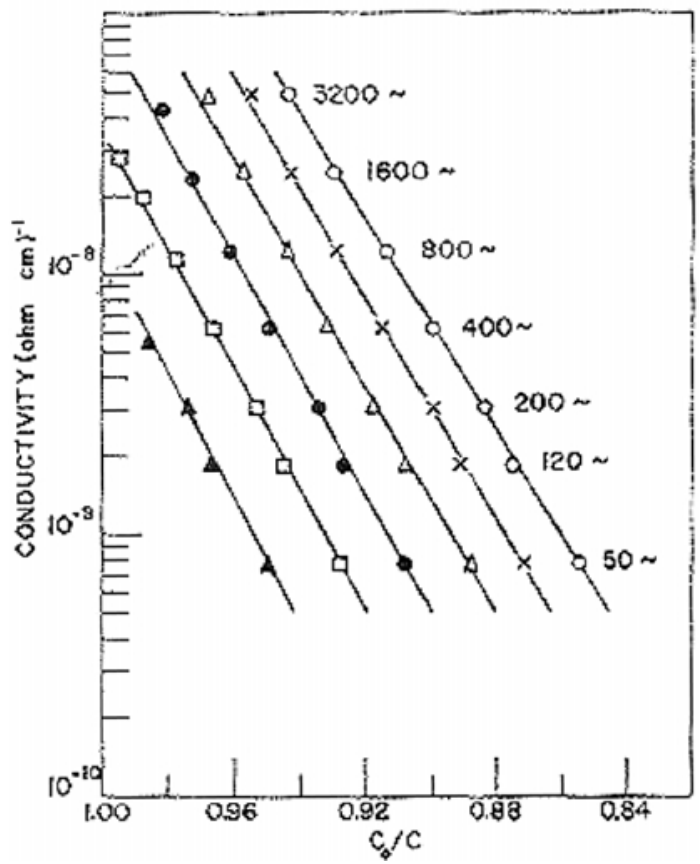

FIGURE 2a Distribution of conductivity in heat-treated, anodized tantalum at several temperatures $(75 \mathrm{~V}$ anodization, heated $30 \mathrm{~min}$ at $400^{\circ}$, in air). $\bigcirc, 30^{\circ} ; \times, 20^{\circ} ; \Delta, 10^{\circ} ; \bullet, 0^{\circ} ; \square,-10^{\circ}$; black $\Delta,-20^{\circ}$.

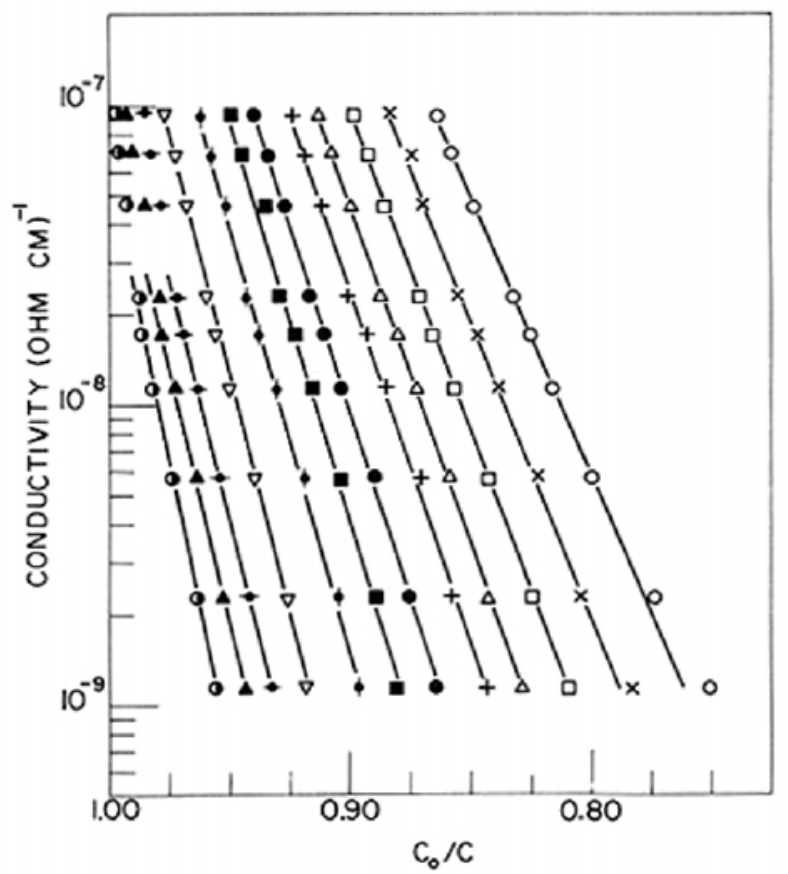

FIGURE $2 \mathrm{~b}$ Conductivity profiles across oxide film of anodized niobium heated in air at $170^{\circ}$ for $30 \mathrm{~min} . \bigcirc^{\circ}, 23.8^{\circ}$; $\times, 12.0^{\circ} ; \square, 2.4^{\circ} ; \Delta,-7.0^{\circ} ;+,-15.0^{\circ} ; \bullet,-26.0^{\circ} ; \mathbf{\square},-35.0^{\circ}$; vertical dash with center dot, $-71.0^{\circ} ;$ black $\Delta$, $-80.0^{\circ}$; half dark circle, $-90^{\circ}$. 
the conditions of these experiments, up to about 20 percent of the dielectric can be shorted out for both systems, resulting in an increase in capacitance of comparable amount.

This "excess" capacitance is dependent on frequency, temperature, and DC bias, to a degree that is undesirable for capacitor applications. The dependence on DC bias results from a depletion of electrons over a thickness of oxide, changing the effective dielectric thickness. The dielectric losses depend on the sharpness of the effective electrode-dielectric interface, so that these gradients of conductivity introduce additional losses.

Note in Figures $2 \mathrm{a}$ and $2 \mathrm{~b}$ that the conductivity levels for similar measurement conditions are about two orders of magnitude higher in the $\mathrm{Nb}_{2} \mathrm{O}_{5}$ films than in the $\mathrm{Ta}_{2} \mathrm{O}_{5}$ films. Moreover, note that the Ta samples were heated at $400{ }^{\circ} \mathrm{C}$ while the damage was done to the $\mathrm{Nb}$ sample at only $170^{\circ} \mathrm{C}$. Clearly the $\mathrm{Nb}$ system is much more sensitive to this kind of thermal processing damage. In the Ta-based capacitors the damage is avoided by keeping the pyrolysis temperature for the application of the $\mathrm{MnO}_{2}$ relatively low, in the range $250-300^{\circ} \mathrm{C}$, and by a subsequent solder annealing step introducing electrochemical oxidation that pumps oxygen into the film faster than the Ta metal is extracting it from the oxide. These remedies have not been fully effective for $\mathrm{Nb}$-based capacitors, which may appear deceptively stable on life-test with applied voltage, since the voltage tends to continuously replenish the oxygen content of the film. However, for high temperature storage without applied bias, problems can rapidly arise.

\section{ROUTES TO A STABLE DIELECTRIC LAYER}

The process of transferring oxygen through the oxide film and into the underlying metal can be broken down into three steps, any one of which could be the slowest and thus be the ratedetermining step: (1) entry of oxygen into the oxide from the ambient atmosphere, (2) diffusion of the oxygen across the oxide film, and (3) crossing the metal-metal oxide interface into the metal. From an atomic and structural point of view, this is more accurately described as the removal of oxygen from the oxide by the metal, leaving the adjacent oxide oxygendeficient; followed by diffusion of this oxygen deficiency, loosely called oxygen vacancies, across the oxide to the outer surface where the vacancies are filled from the ambient air. For the well-studied $\mathrm{Ta} / \mathrm{Ta}_{2} \mathrm{O}_{5}$ system, heat-treated at $400{ }^{\circ} \mathrm{C}$ in air $[2,3]$, the percentage increase in capacitance is roughly independent of film thickness, at about 20 percent, for anodization voltages above about 40 volts. This suggests that the compositions of the oxide at the interfaces are fixed at equilibrium values, and that diffusion across the film is the ratedetermining step. In this case the conductivity gradient decreases with increasing oxide thickness and intersects the $s_{0}$ level at the same percentage of the film thickness. However, the percentage increase in capacitance resulting from heat-treatment decreases with decreasing film thickness for anodization voltages below 40 volts, and is essentially zero for 10 volt films. This implies that the diffusion in the thinner films has become faster than the exchange reaction at the metal-metal oxide interface. Thus the oxygen content is maintained at a high value across the entire film thickness.

The use of nitrogen to affect the migration of oxygen at the dielectric interface in both Ta and $\mathrm{Nb}$ is well-documented [4,5]. Capacitors made from anode pellets sintered from such powders show improved stability relative to the untreated metal. For example, capacitors formed on thin films of Ta containing 13 to 27 atomic percent nitrogen were studied $[5,6]$ as a function of depth. An Auger spectroscope was used to study composition as a function of depth within the film. The results are show in Figure 3, and indicate a uniform N concentration in about 80 percent of the film thickness adjacent to the Ta. 


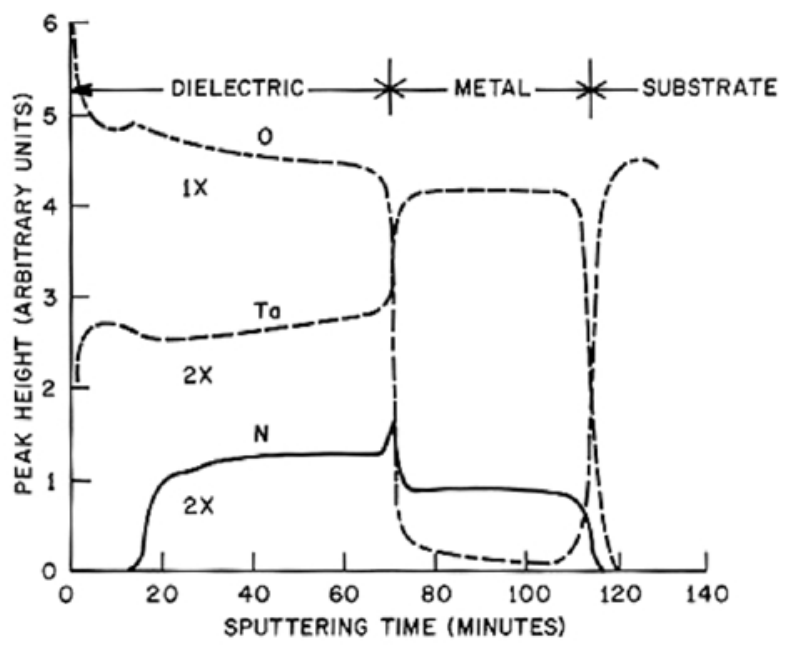

FIGURE 3 Peak height of Ta, O, and N Auger signals as a function of sputtering time to illustrate distribution of these elements in anodic films formed to $150 \mathrm{~V}$ on Ta-13 atomic $\% \mathrm{~N}$ alloy film.

The outer 20 percent contained no detectable N. This duplex film results from the simultaneous transport of $\mathrm{Ta}^{+5}$ and $\mathrm{O}^{-2}$ ions through the film during the growth process $[7,8]$. That part of the film that grows at the metal-metal oxide interface due to oxygen transport incorporates $\mathrm{N}$ from the nitrided $\mathrm{Ta}$, while that part of the film that grew at the electrolyte interface due to Ta transport remained free of $\mathrm{N}$. The incorporated $\mathrm{N}$ is apparently immobile in the oxide film. It was also noted that the dielectric constant of the film decreased by about half as the $\mathrm{N}$ content increased to 27 atomic percent $[5,6]$. When Ta is anodized in aqueous solutions of phosphoric acid, the outer part of the film, formed by the diffusion of $\mathrm{Ta}^{+5}$ to the electrolyte interface, contains phosphorus, while the inner part of the film remains phosphorus-free $[7,8]$. It has been shown that phosphorus suppresses the diffusion of oxygen in anodic $\mathrm{Ta}_{2} \mathrm{O}_{5}$ films [9]. Since at steady state the diffusion rate, the product of the diffusion constant times the concentration gradient, must be constant throughout the film, the concentration gradient, and hence the conductivity gradient in the heat-treated films breaks up into two slopes, as shown in Figure 4 [8].

The shallow gradient is in the inner, P-free, region, and this is undesirable. The shallow gradient in the critical region near $\sigma_{0}$ causes an increase in the temperature, frequency, and bias dependency of capacitance, as well as higher dielectric losses. If $\mathrm{N}$ incorporated into the inner part of the oxide film formed on nitrided $\mathrm{Nb}$ has a similar effect on the oxygen diffusion rate, a more favorable situation is achieved. The steeper gradient in the vicinity of $\sigma_{0}$ will give improved properties.

Studies to date indicate that nitriding $\mathrm{Nb}$ has a similar effect on finished capacitor stability as nitriding Ta. This is likely due to the reduction in the tendency of the metal to extract oxygen from the oxide film, thus maintaining a stable composition at the interface and minimizing diffusion.

However, addition of nitrogen is not without cost in capacitor performance. Figure 5 shows the $\mathrm{CV}$ of $\mathrm{Nb}$ capacitor nitrided to various levels. The data show a reduction in the bias dependence of capacitance suggesting improved interfacial stability, but at the expense of lower capacitance, either $\mathrm{CV} / \mathrm{g}$ or $\mathrm{CV} / \mathrm{cc}$, due to the significantly lower dielectric constant.

Another approach to achieving a stable $\mathrm{Nb}$-based capacitor is to replace the $\mathrm{Nb}$ metal anode with $\mathrm{NbO}$, as reported by the authors and a recent patent publication $[10,11]$. High 


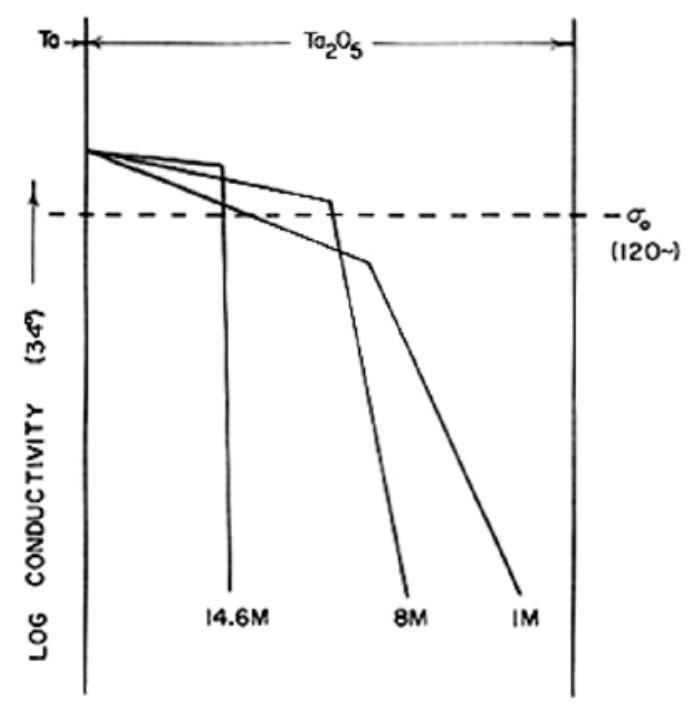

FIGURE 4 Schematic representation of the conductivity profiles across oxide films on tantalum anodized in various concentrations of $\mathrm{H}_{3} \mathrm{PO}_{5}$, and heat-treated at $400^{\circ}$ in air.

quality $\mathrm{Nb}_{2} \mathrm{O}_{5}$ dielectric films are produced on $\mathrm{NbO}$ by standard electrochemical techniques, with only minor modification of the process used to make Ta-based capacitors.

$\mathrm{NbO}$ is a stable compound with a metallic conductivity just one order of magnitude less than $\mathrm{Nb}$ metal, but well within the requirements of a capacitor material.

Combined with the metal-like electrical properties are its ceramic-like physical properties of high hardness and bulk diffusion sintering behavior. The use of $\mathrm{NbO}$ for capacitors offers the opportunity for improved stability by reducing the oxygen gradient across the dielectric interface, thus reducing the driving force for oxygen diffusion into the bulk conductor. This is demonstrated by the data shown in Figure 6, which shows the change in capacitance (measured in liquid electrolyte) as a function of annealing temperature.



FIGURE 5 Behaviour of nitrided $\mathrm{Nb}$ anodes (annealed at $350{ }^{\circ} \mathrm{C} / 10 \mathrm{~min}$ ). 


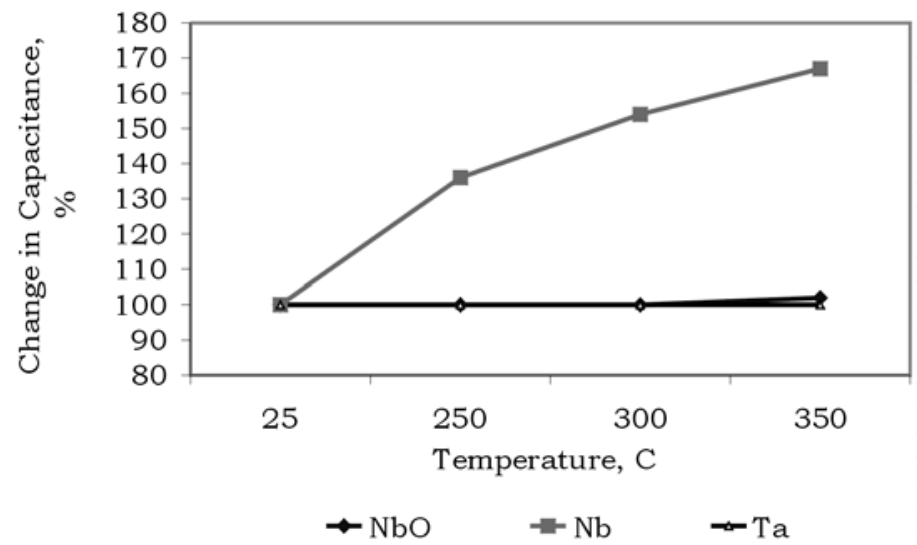

FIGURE $6 \mathrm{Ta}, \mathrm{Nb}$, and $\mathrm{NbO}$ anodes during annealing.

These anodes were anodized to $40 \mathrm{~V}$ and were later subjected to various annealing temperatures. Since capacitance change is believed to relate to the stability of the substrate/ dielectric interface, an increase in measured capacitance indicates a thinning of the dielectric due to loss of oxygen. The greater the loss, the greater the increase in capacitance. The data show that capacitance change of $\mathrm{Nb}$ anodes is significant, while changes in $\mathrm{NbO}$ and tantalum are comparable, and much more limited. This indicates improved stability of the dielectric layer for $\mathrm{NbO}$ versus niobium, which can be attributed to the fact that high concentration of oxygen already present in the $\mathrm{NbO}$ substrate.

It is also observed that $\mathrm{Ta}$ metal wires embedded in $\mathrm{NbO}$ anodes are still flexible after sintering, suggesting that oxygen diffusion from $\mathrm{NbO}$ to $\mathrm{Ta}$ is very limited. Ta wires will become brittle if a significant amount of oxygen migrates from the bulk material into the wire.

It should be noted that an improved substrate/dielectric interface in $\mathrm{NbO}$ capacitor does not affect the dielectric constant. This is a key advantage of $\mathrm{NbO}$ over nitrided $\mathrm{Nb}$ metal, thus offering greater capacitance for a powder of the same surface area. In order to evaluate the impact of manganizing process, $\mathrm{NbO}$ anodes were either sequentially exposed to $300^{\circ} \mathrm{C}$ (10 minutes) 20 times or manganized using standard tantalum process. Only minimum bias dependency was observed in both cases, as shown in Figures 7 and 8.

Reliability evaluation, including $85^{\circ} \mathrm{C}$ life test, high temperature storage test (without applied voltage), and Weibull analysis, has demonstrated that $\mathrm{NbO}$ capacitors have stable

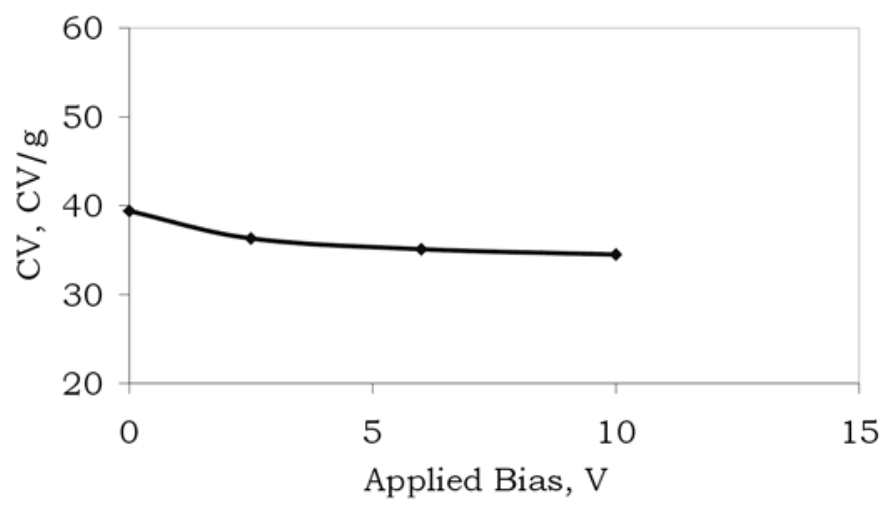

FIGURE 7 Bias dependency of $\mathrm{NbO}$ anodes after 20 exposure to $300^{\circ} \mathrm{C} / 10 \mathrm{~min}$. 


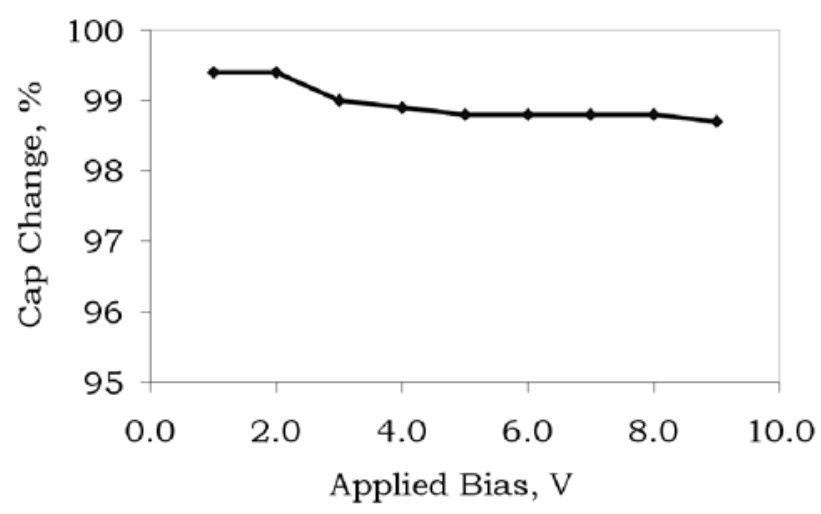

FIGURE 8 Bias dependency of $\mathrm{MnO}_{2}$ coated $\mathrm{NbO}$ parts $(150 \mathrm{uF} / 10 \mathrm{~V})$.



FIGURE 9 Oxidation energy of materials.

properties at elevated temperature through life testing. NbO capacitors also have very low DC leakage current, approaching that of its Ta counterparts.

Another major advantage of $\mathrm{NbO}$ is that it is much less pyrophoric than its metal counterparts due to its partially oxidized nature. It requires much more energy to ignite and burns more slowly. Figure 9 compares the combustion energy of $\mathrm{NbO}, \mathrm{Nb}$, and $\mathrm{Ta}$. The calculated values are based on available thermal dynamic data while the experiment values were

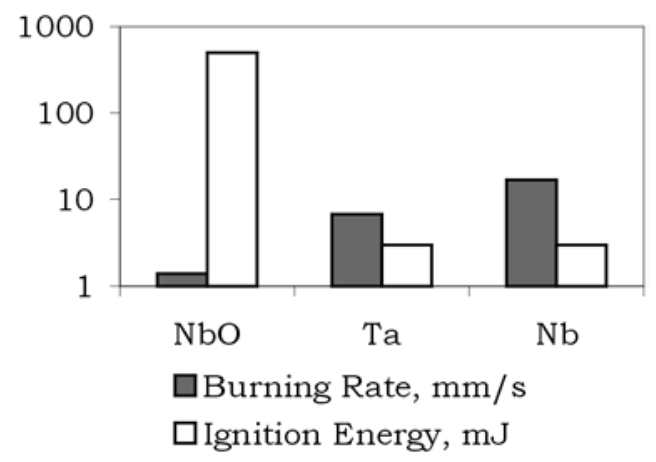

FIGURE 10 Materials flammability. 
measured using differential scanning calorimetry (DSC). As expected, $\mathrm{NbO}$ produces only half the heat of $\mathrm{Nb}$ and $\mathrm{Ta}$.

Figure 10 summarizes the results of ignition energy and burning rate studies. Compared to $\mathrm{Nb}$ and Ta powders, $\mathrm{NbO}$ requires at least an order of magnitude more energy to ignite, and burns much more slowly.

It is obvious that $\mathrm{NbO}$ powder will have lower flammability potential than $\mathrm{Nb}$ or Ta metal powders in capacitor manufacturing operations. We also believe $\mathrm{NbO}$ capacitors will offer significant benefits in situations where ignition potential is a concern.

\section{CONCLUSIONS}

The successful manufacture of niobium based solid electrolyte capacitors requires that the $\mathrm{Nb} / \mathrm{Nb}_{2} \mathrm{O}_{5}$ interface be stabilized so that changes in conductivity within and near the interface do not change with time or temperature. There are currently two approaches that have been examined for their effect on long term stability. One is to nitride the $\mathrm{Nb}$ powder, as reported earlier for tantalum, so that the inner portion of the dielectric film incorporates $\mathrm{N}$. This has been shown to increase interfacial stability by either retarding the migration of oxygen through the film or reducing the tendency of the $\mathrm{Nb}$ to extract oxygen from the oxide. But there is an important side effect in that the dielectric constant of the material is significantly reduced. The other is to replace $\mathrm{Nb}$ metal with $\mathrm{NbO}$, a highly conducting suboxide of $\mathrm{Nb}$. In this case it is believed that the tendency of the anode material to extract oxygen from the $\mathrm{Nb}_{2} \mathrm{O}_{5}$ film has been strongly suppressed. $\mathrm{NbO}$ capacitors have low DC leakage and life testing data similar to that achieved with commercial tantalum capacitors.

\section{References}

[1] Smyth, D. M. and Tripp, T. B. (1966). J. Electrochem. Soc., 113, 1048.

[2] Smyth, D. M., Shirn, G. A. and Tripp, T. B. (1963). J. Electrochem. Soc., 110, 1264.

[3] Smyth, D. M., Shirn, G. A. and Tripp, T. B. (1964). J. Electrochem. Soc., 111, 1331.

[4] Fife, J. (2000). US Patent \#6051044; Chang, H. (1995). US Patent \#5448447.

[5] Tripp, T. B., Creasi, R. M. and Cox, B. (2000). Proceedings, CARTS 2000, p. 82.

[6] Simmons, R. T., Morzenti, P. T., Smyth, D. M. and Gerstenberg, D. (1974). Thin Solid Films, $23,75$.

[7] Randall, J. J., Bernard, W. J. Jr. and Wilkinson, R. R. (1965). Electrochem. Acta, 10, 183.

[8] Smyth, D. M., Tripp, T. B. and Shirn, G. A. (1966). J. Electrochem. Soc., 113, 100.

[9] Pawel, R. E. and Campbell, J. J. (1966). J. Electrochem. Soc., 113, 1204.

[10] Qiu, Y., Kitchell, R., Smyth, D. and Kimmel, J. (2001). Proceedings, CARTS 2001, p. 99.

[11] Fife, J. International Published Application No. WO 00/15555. 

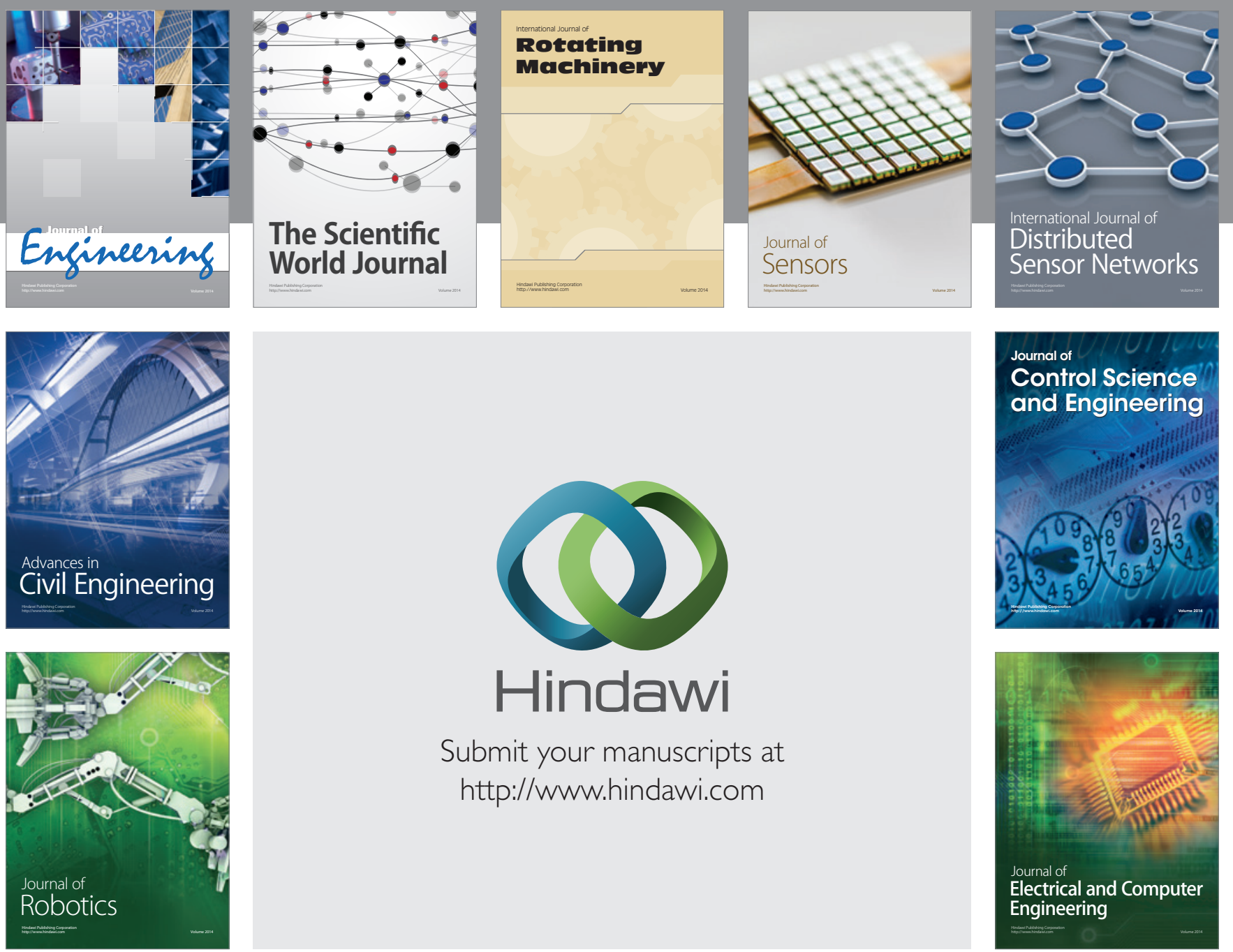

Submit your manuscripts at

http://www.hindawi.com
